This item was submitted to Loughborough's Research Repository by the author.

Items in Figshare are protected by copyright, with all rights reserved, unless otherwise indicated.

\title{
Pressure work and viscous dissipation in the equations of thermal convection in a vertical channel
}

\section{PLEASE CITE THE PUBLISHED VERSION}

http://dx.doi.org/10.1007/s10665-016-9876-4

\section{PUBLISHER}

(c) Springer Verlag

\section{VERSION}

AM (Accepted Manuscript)

\section{PUBLISHER STATEMENT}

This work is made available according to the conditions of the Creative Commons Attribution-NonCommercialNoDerivatives 4.0 International (CC BY-NC-ND 4.0) licence. Full details of this licence are available at: https://creativecommons.org/licenses/by-nc-nd/4.0/

\section{LICENCE}

CC BY-NC-ND 4.0

\section{REPOSITORY RECORD}

Kay, Anthony. 2019. "Pressure Work and Viscous Dissipation in the Equations of Thermal Convection in a Vertical Channel”. figshare. https://hdl.handle.net/2134/22423. 


\section{Pressure work and viscous dissipation in the equations of thermal convection in a vertical channel}

Anthony Kay.

Received: date / Accepted: date

Abstract Equations for fully developed flow in a vertical channel have been solved, taking into account viscous dissipation, and using formulations with and without pressure work. Perturbation solutions are used to distinguish the effects of viscous dissipation from pressure work. Viscous dissipation has very little effect on free convection flows driven by temperature differences or heat fluxes at the channel walls, but it may play a major role in forced convection.

Keywords Thermal convection - Vertical channel · Viscous dissipation · Pressure work

\section{Introduction}

In a recent paper [1] the present author solved equations for forced convection in a Newtonian fluid confined between parallel vertical plates held at equal temperatures. In the energy equation, temperature changes in the fluid were accounted for by heat conduction, viscous dissipation and pressure work. Solutions were presented both with and without the inclusion of pressure work, for the full range of applied pressure gradients for which solutions exist. The results were of some mathematical interest, but many of the interesting features were outside the range of parameter space that is physically accessible or of practical relevance in engineering. With any given value of applied pressure gradient, there exist either dual solutions or no solution at all; in particular, with zero applied pressure gradient, one solution branch yields static fluid with no temperature variation, whereas the second solution branch yields

Anthony Kay

Department of Mathematical Sciences, Loughborough University, Loughborough, Leicestershire, LE11 3TU, United Kingdom

Tel.: +44-1509-222878

E-mail: A.Kay@lboro.ac.uk 
"passive convection", in which viscous dissipation provides the heat to maintain (through buoyancy forces) the flow that gives rise to the dissipation. Setting up the flow specified by this second solution branch would be very difficult, and indeed Miklavčič [2] has recently shown that solutions on this branch are unstable. Even on the stable first branch, flow velocities and temperature gradients are only of reasonable magnitude if the applied pressure gradient is of moderate magnitude; furthermore, large applied pressure gradients may violate the Boussinesq approximation (made in all the calculations) which requires any vertical dynamic pressure gradient to be small compared to the hydrostatic pressure gradient. Hence it would be sensible to focus more attention on first-branch solutions in the regime of smaller applied pressure gradient.

The issue of the relative importance of viscous dissipation and pressure work in convection flows was discussed as long ago as 1971 by Kuiken [3], but has received more attention recently, including in the context of flows in vertical ducts. Of particular interest is the consistency of neglecting or retaining either of these terms in the energy equation when the Boussinesq approximation is made in the momentum equation. For boundary-layer flow adjacent to a heated vertical plate, Kuiken [3] found that pressure work may be important whereas viscous dissipation would be no larger than effects neglected in the Boussinesq approximation. Turcotte et al. [4] have shown that pressure work and viscous dissipation are proportional to the same dimensionless parameter in Bénard convection, so the two effects must be considered simultaneously. Barletta $[5,6]$ has discussed the relative merits of the enthalpy formulation and the internal-energy formulation of the energy equation: in the latter, there is no pressure work term because the Boussinesq approximation implies that the velocity is solenoidal, and this formulation is found to be preferable as long as care is taken to select the correct specific heat for the fluid under consideration. For flow in a lid-driven square cavity, Barletta and Nield [7] find that a formulation including viscous dissipation but neglecting pressure work gives more reasonable results than when both effects are explicitly included. On the other hand, when investigating flow in a porous medium in a vertical channel, the same authors [8] find that pressure work is important, in particular producing a cooling effect in upward flow greater than the heating due to viscous dissipation. A similar effect was found by Kay [1] in forced convection of a Newtonian fluid in a vertical channel when the forcing is relatively small; as in the discussion of Rayleigh-Bénard convection by Barletta and Nield [9], it was noted that pressure work is linear in the vertical velocity whereas viscous dissipation is quadratic, so that pressure work must have greater magnitude in the limit of small velocities.

Where previous studies of free or forced convection in vertical channels have considered the effect of viscous dissipation, pressure work has in most cases been neglected. This has been done both with boundary conditions of fixed wall temperatures, e.g. [10] and with fixed heat fluxes, e.g. [11]. However, from the discussion above, it appears that the neglect of pressure work may not be justified, particularly in the important case of small or moderate flow 
velocities. The object of the present study is to compare results obtained using formulations both with and without pressure work, but including viscous dissipation in all cases. We shall use perturbation expansions with the forcing as the small parameter, whether that be the applied pressure gradient for the case of pure forced convection, or a temperature difference or heat flux between the walls for the case of pure free convection (we do not consider the more complicated case of mixed convection). As well as being appropriate for small values of the forcing, perturbation expansions will give more insight than numerical solutions into how the effects of viscous dissipation and pressure work come into play.

\section{Governing equations}

A thorough discussion of the governing equations for convection in vertical ducts was given in [1], and the reader is referred there for the derivation of the equations which follow. We consider only steady, fully developed flow of a Newtonian fluid in a channel of width $2 L$ between parallel, vertical walls located at $Y= \pm L$. The coefficient of thermal expansion $\beta$ is assumed to be constant, so that the density $\rho$ is linearly related to the temperature $T$,

$$
\rho=\rho_{m}\left(1-\beta\left(T-T_{m}\right)\right)
$$

where the reference temperature $T_{m}$ and reference density $\rho_{m}$ are required to be the cross-section means of these quantities $[1,12]$. Other fluid properties that are also assumed constant are viscosity $\mu$, thermal conductivity $k$ and specific heat $c$.

The momentum equation is a balance between the applied pressure gradient (which must be uniform in fully developed flow), the buoyancy force and the viscous stress. Under the Boussinesq approximation it takes the form

$$
-\frac{\mathrm{d} P_{d}}{\mathrm{~d} Z}+\rho_{m} g \beta\left(T-T_{m}\right)+\mu \frac{\mathrm{d}^{2} W}{\mathrm{~d} Y^{2}}=0,
$$

where the dynamic pressure $P_{d}$ is the difference between the total pressure and the hydrostatic pressure, and the velocity $W$ is in the vertical $(Z)$ direction.

The energy equation may be formulated with or without a term for pressure work:

$$
c \rho_{m} \frac{\mathrm{d} T}{\mathrm{~d} t}=-\beta T_{m} \rho_{m} g W+\mu\left(\frac{\mathrm{d} W}{\mathrm{~d} Y}\right)^{2}+k \frac{\mathrm{d}^{2} T}{\mathrm{~d} Y^{2}}
$$

or

$$
c \rho_{m} \frac{\mathrm{d} T}{\mathrm{~d} t}=\mu\left(\frac{\mathrm{d} W}{\mathrm{~d} Y}\right)^{2}+k \frac{\mathrm{d}^{2} T}{\mathrm{~d} Y^{2}} .
$$

The pressure work term in (3) has been formulated consistently with the Boussinesq approximation, assuming that the dynamic pressure gradient can be neglected in comparison with the hydrostatic pressure gradient in this term, and using the cross-section mean value of temperature. We have deliberately 
been ambiguous about the definition of the specific heat $c$ (whether at constant pressure, or constant volume, etc.). Barletta [5,6] discusses this issue in some detail, but when the equations are nondimensionalised the difference between the values of $c$ appropriate for (3) or (4) disappears from the equations. Our objective is to compare the flow and thermal conditions predicted according to the two formulations, and the differences we shall find cannot be reconciled by returning to dimensioned variables and inserting different values of specific heat.

For purely vertical flow,

$$
\frac{\mathrm{d} T}{\mathrm{~d} t}=\frac{\partial T}{\partial t}+W \frac{\partial T}{\partial Z}
$$

but steady solutions for the flow velocity are admitted if both the temporal and downstream variations of temperature are constant in space and time. We then write

$$
H=c \rho_{m} \frac{\partial T}{\partial t} \quad \text { and } \quad G=c \rho_{m} \frac{\partial T}{\partial Z},
$$

with $H$ and $G$ assumed constant. We can now use the momentum equation (2) to eliminate the temperature from either form of the energy equation, yielding

$$
H+W G=-\beta T_{m} \rho_{m} g W+\mu\left(\frac{\mathrm{d} W}{\mathrm{~d} Y}\right)^{2}-\frac{\mu k}{\rho_{m} g \beta} \frac{\mathrm{d}^{4} W}{\mathrm{~d} Y^{4}}
$$

or

$$
H+W G=\mu\left(\frac{\mathrm{d} W}{\mathrm{~d} Y}\right)^{2}-\frac{\mu k}{\rho_{m} g \beta} \frac{\mathrm{d}^{4} W}{\mathrm{~d} Y^{4}} .
$$

However, solutions with $\mathrm{d} T / \mathrm{d} t=0$, i.e. with the left-hand sides of (7) and (8) set to zero, are admitted unless the boundary conditions specify the heat flux at both walls.

The half-width $L$ of the channel provides the obvious scale for defining the dimensionless cross-stream co-ordinate,

$$
y=\frac{Y}{L} .
$$

It is less obvious how dimensionless velocities and temperatures should be defined. In [1] velocity and temperature scales were defined from a balance between heat conduction and viscous dissipation. While we are not expecting viscous dissipation to be a leading-order effect in most cases, a nondimensionalisation based on these scales does have the virtue that it can be used in every scenario to be considered below, whether for free or forced convection, and whether temperatures or heat fluxes are specified at walls. Hence we shall use the same dimensionless variables and parameters as in [1], while being careful about the interpretation of large or small values of these variables appearing in solutions of our equations. The dimensionless vertical velocity and temperature are

$$
w=\frac{\rho_{m} g \beta L^{2}}{k} W, \quad \theta=\frac{\left(\rho_{m} g \beta L^{2}\right)^{2}}{\mu k}\left(T-T_{m}\right) .
$$


The dimensionless dynamic pressure gradient (in forced convection) is

$$
\lambda=\frac{\rho_{m} g \beta L^{4}}{\mu k} \frac{\mathrm{d} P_{d}}{\mathrm{~d} Z},
$$

and the dimensionless horizontal heat flux is

$$
q=-\frac{\left(\rho_{m} g \beta\right)^{2} L^{5}}{\mu k} \frac{\partial T}{\partial y} .
$$

Dimensionless temporal and streamwise temperature variations (when heat flux is specified at both walls) are

$$
\eta=\frac{\left(\rho_{m} g \beta L^{3}\right)^{2}}{\mu k^{2}} H=\frac{\left(\rho_{m} g \beta L^{3}\right)^{2}}{\mu k^{2}} c \rho_{m} \frac{\mathrm{d} T}{\mathrm{~d} t}, \quad \gamma=\frac{\rho_{m} g \beta L^{4}}{\mu k} G=\frac{c \rho_{m}^{2} g \beta L^{4}}{\mu k} \frac{\mathrm{d} T}{\mathrm{~d} Z},
$$

and a dimensionless parameter introduced by Schneider [13] to compare the magnitudes of pressure work and viscous dissipation is

$$
N=\frac{\left(\rho_{m} g \beta L^{2}\right)^{2} T_{m}}{\mu k}
$$

In terms of these dimensionless variables, equation (7) becomes

$$
-\frac{\mathrm{d}^{4} w}{\mathrm{~d} y^{4}}+\left(\frac{\mathrm{d} w}{\mathrm{~d} y}\right)^{2}-N w=\eta+\gamma w .
$$

This is the equation that we need to solve, with and without the pressure work term $N w$; the terms on the right-hand side will be omitted unless the boundary conditions specify heat flux on both walls. The dimensionless form of the momentum equation (2) is

$$
-\lambda+\theta+\frac{\mathrm{d}^{2} w}{\mathrm{~d} y^{2}}=0,
$$

and is required only to diagnose the temperature once we have solved for the vertical velocity.

We shall be considering four scenarios: free convection or forced convection, each with temperatures or heat fluxes specified on the walls. Two boundary conditions apply on the walls in all cases: the no-slip condition, $w=0$ at each wall, and the requirement for any dynamic pressure gradient to be balanced by the total wall shear stress on the two walls, where dimensionless shear stress is $\mathrm{d} w / \mathrm{d} y$. Other boundary conditions differ between the cases, as specified below.

1. Forced convection, fixed wall temperatures.

For the convection to be purely forced, the wall temperatures must be equal. We then have symmetry about the mid-plane of the duct, so symmetry conditions are imposed at $y=0$ instead of wall conditions at $y=-1$. Thus we solve

$$
-\frac{\mathrm{d}^{4} w}{\mathrm{~d} y^{4}}+\left(\frac{\mathrm{d} w}{\mathrm{~d} y}\right)^{2}-N w=0
$$


(including pressure work) or

$$
-\frac{\mathrm{d}^{4} w}{\mathrm{~d} y^{4}}+\left(\frac{\mathrm{d} w}{\mathrm{~d} y}\right)^{2}=0
$$

(omitting pressure work), subject to the boundary conditions

$$
w^{\prime}(0)=0, \quad w^{\prime \prime \prime}(0)=0, \quad w(1)=0, \quad w^{\prime}(1)=\lambda,
$$

in which primes indicate derivatives with respect to $y$. The last condition in (19) is the balance of dynamic pressure gradient with wall shear stress: we seek perturbation solutions valid in the limit $\lambda \rightarrow 0$, i.e. for small values of the dimensionless pressure gradient. Note that no temperature boundary conditions are applied in solving (18); the symmetry condition is sufficient to ensure that the walls at $y= \pm 1$ have equal temperatures [1]. Dimensionless temperatures $\theta$ (relative to the cross-section mean) are obtained from (16) after solving for $w$; however, since the wall temperature $\theta_{w}$ is supposed to be known, we present formulae and plots for $\theta-\theta_{w}$ (rather than $\theta$ ) in Section 3.1 below, to give temperatures relative to this fixed value.

2. Forced convection, insulated walls.

For purely forced convection with heat flux specified at the walls, that heat flux must be zero; note that dimensionless heat flux is $-\partial \theta / \partial y=\partial^{3} w / \partial y^{3}$, from (16). Again there is symmetry about the mid-plane, with conditions at $y=0$ as in case 1 . Thus we solve

$$
-\frac{\mathrm{d}^{4} w}{\mathrm{~d} y^{4}}+\left(\frac{\mathrm{d} w}{\mathrm{~d} y}\right)^{2}-N w=\eta+\gamma w
$$

(including pressure work) or

$$
-\frac{\mathrm{d}^{4} w}{\mathrm{~d} y^{4}}+\left(\frac{\mathrm{d} w}{\mathrm{~d} y}\right)^{2}=\eta+\gamma w
$$

(omitting pressure work), subject to the boundary conditions

$$
w^{\prime}(0)=0, \quad w^{\prime \prime \prime}(0)=0, \quad w(1)=0, \quad w^{\prime}(1)=\lambda, \quad w^{\prime \prime \prime}(1)=0,
$$

again seeking perturbation solutions valid in the limit $\lambda \rightarrow 0$. There are five boundary conditions for a fourth-order equation, but since neither $\eta$ nor $\gamma$ is known a priori the system is in fact under-determined. This is because no account has been taken of entry or exit conditions, which would determine any streamwise temperature gradient $\gamma$. We shall discuss this issue in more detail when considering solutions of these equations in Section 3.2 below, where formulae and plots for temperature will be given relative to the cross-section mean (i.e. simply as $\theta$ ), since the temperature at any cross-stream location is varying at a constant rate with time and/or downstream position. 
3. Free convection, fixed wall temperatures.

Free convection means that the dynamic pressure gradient $\lambda$ is zero, so that (16) becomes

$$
\theta=-\partial^{2} w / \partial y^{2}
$$

With viscous dissipation included, we cannot assume antisymmetry: the mid-plane temperature will not be the mean of the wall temperatures, and the mid-plane velocity may not be zero. Thus, with a given dimensionless temperature difference $\Theta$ between the walls, we solve (17) or (18) with the boundary conditions

$$
w(-1)=0, \quad w(1)=0, \quad w^{\prime}(1)-w^{\prime}(-1)=0, \quad w^{\prime \prime}(1)-w^{\prime \prime}(-1)=-\Theta
$$

in which the third condition specifies zero total wall shear stress. We seek perturbation solutions valid in the limit $\Theta \rightarrow 0$. Wall temperatures are supposed known, so in Section 3.3 we shall present formulae and plots for $\theta-\theta_{M}$ where $\theta_{M}$ is the (fixed) mean of the temperatures at the two walls, not in general the same as the cross-section mean.

4. Free convection, fixed wall heat fluxes.

We consider only the case where the dimensionless heat flux $q$ into the channel at the wall $y=1$ is equal to the heat flux out of the channel at the wall $y=-1$. We shall see that this case with antisymmetric boundary conditions is in some ways unrepresentative of free convection driven by wall heat flux; however, it is the case that is most comparable to free convection driven by unequal wall temperatures (case 3 above). A full exploration of different heat flux boundary conditions is beyond the scope of this paper.

As with the fixed-temperature free convection case, we cannot assume that velocity and temperature profiles are antisymmetric. Thus we solve (20) or (21) with boundary conditions

$$
w(-1)=0, w(1)=0, w^{\prime}(1)-w^{\prime}(-1)=0, w^{\prime \prime \prime}(-1)=-q, w^{\prime \prime \prime}(1)=-q,
$$

seeking perturbation solutions valid in the limit $q \rightarrow 0$. Comments made in case 2 regarding the number of boundary conditions and the presentation of formulae and plots for temperatures apply here also.

Before proceeding to the solutions, we consider what magnitudes of physical parameters are allowed in order to fulfil both the mathematical requirement of validity of the perturbation solutions and the physical requirement that the Boussinesq approximation be applicable. For this purpose, the parameter $N$, originally introduced as a dimensionless measure of the importance of pressure work, turns out to be useful independently of whether we are using the formulation including or excluding pressure work. We first introduce alternative dimensionless forms of the forcing parameters, which may be considered more intuitive than $\lambda, \Theta$ and $q$, and which are related to them as follows. For forced convection, an intuitive nondimensionalisation of the dynamic pressure 
gradient is with respect to the hydrostratic pressure gradient:

$$
\frac{\mathrm{d} P_{d}}{\mathrm{~d} Z} / \frac{\mathrm{d} P_{h}}{\mathrm{~d} Z}=\frac{\mu k}{\left(\rho_{m} g\right)^{2} \beta} L^{-4} \lambda=\frac{\beta T_{m}}{N} \lambda .
$$

For free convection, a temperature difference $\Delta T$ between the walls could be nondimensionalised with respect to the absolute mean temperature:

$$
\frac{\Delta T}{T_{m}}=\frac{1}{N} \Theta,
$$

and a corresponding nondimensionalisation of heat flux would be

$$
\frac{-[\partial T / \partial y]_{\text {wall }}}{T_{m} / L}=\frac{1}{N} q .
$$

Clearly each of the parameters on the left-hand sides of (26) - (28) must be small compared to unity in order for the Boussinesq approximation to be valid, while the parameters $\lambda, \Theta$ or $q$ need to be small in some sense for the perturbation solutions to be valid. If we fix $\lambda, \Theta$ or $q$ to fulfil the latter requirement, then from (26) - (28) a large value of $N$ will guarantee that the former is satisfied, while a small value of $N$ may restrict the range of allowable values of $\lambda, \Theta$ or $q$.

Once we have chosen a fluid and fixed its mean temperature $T_{m}$ (so that fluid parameters $\rho_{m}, \beta, \mu$ and $k$ are also fixed), $N$ depends only on the duct width: for example, for water at $T_{m}=20^{\circ} \mathrm{C}=293 \mathrm{~K}$, we find $N \approx 2.1 \times$ $10^{6} \mathrm{~L}^{4}$ while for glycerine (chosen for illustration since high viscosity is likely to render viscous dissipation more prominent) at the same temperature, $N \approx$ $2.8 \times 10^{4} L^{4}$, with $L$ in metres. Our solutions in Section 3 for the formulation including pressure work will be plotted for the parameter values $N=4$ and $N=4 \times 10^{4}$, corresponding respectively to $L=3.7 \mathrm{~cm}$ and $L=37 \mathrm{~cm}$ for water, or $L=11 \mathrm{~cm}$ and $L=1.1 \mathrm{~m}$ for glycerine. The larger value of $N$, with its implication of duct dimensions only found in very large industrial or possibly geophysical applications, will at least guarantee that the Boussinesq approximation is applicable; and the large- $N$ regime is also of mathematical interest.

In water at $20^{\circ} \mathrm{C}, \beta T_{m} \approx 0.061$ while in glycerine at the same temperature, $\beta T_{m} \approx 0.132$. Thus for forced convection with $\lambda$ of order unity, the ratio of dynamic to hydrostatic pressure gradient in (26) will be very small with $N=4 \times 10^{4}$ and moderately small with $N=4$. We shall actually plot solutions with $\lambda=10$, which will be seen to still yield valid perturbation expansions but is stretching the validity of the Boussinesq approximation if $N=4$. For free convection, equations (27) and (28) imply that values of $\Theta$ or $q$ should be small compared to $N$; we shall plot solutions with $\Theta=10$ and with $q=10$, but with $N=400$ rather than $N=4$ as in the forced convection cases. Note that the value of 10 taken for the forcing parameters $\lambda, \Theta$ and $q$ can be considered "small" in the context of perturbation expansions because higher-order terms in those expansions will all be found to contain numerical 
constants much greater than 10 in their denominators. In all cases, the requirement for sufficiently large $N$ to retain the Boussinesq approximation should be interpreted as a minimum allowable duct width, and remains in place when we use the formulation excluding pressure work.

\section{Perturbation solutions}

3.1 Forced convection with fixed wall temperatures

We seek solutions of (17) and (18) in the form of expansions in powers of the dimensionless dynamic pressure gradient $\lambda$,

$$
\begin{gathered}
w=w_{0}+\lambda w_{1}+\lambda^{2} w_{2}+\ldots, \\
\theta=\theta_{0}+\lambda \theta_{1}+\lambda^{2} \theta_{2}+\ldots
\end{gathered}
$$

At $O(1)$, the last of the boundary conditions (19) yields $w_{0}^{\prime}(1)=0$ which, together with the other boundary conditions, ensures that both (17) and (18) have only the trivial solution $w_{0} \equiv 0$ at this order. At $O(\lambda)$, the formulation (18) without pressure work then yields

$$
\frac{\mathrm{d}^{4} w_{1}}{\mathrm{~d} y^{4}}=0
$$

whereas when pressure work is included we obtain

$$
\frac{\mathrm{d}^{4} w_{1}}{\mathrm{~d} y^{4}}+N w_{1}=0
$$

The boundary conditions in both cases are

$$
w_{1}^{\prime}(0)=0, \quad w_{1}^{\prime \prime \prime}(0)=0, \quad w_{1}(1)=0, \quad w_{1}^{\prime}(1)=1,
$$

and from (16) the temperature perturbation at $O(\lambda)$ is given by

$$
\theta_{1}=1-\frac{\mathrm{d}^{2} w_{1}}{\mathrm{~d} y^{2}}
$$

The solution of (31) with boundary conditions (33) is

$$
w_{1}=-\frac{1}{2}\left(1-y^{2}\right)
$$

so $\theta_{1}=0$ from (34). Thus we have a simple Poiseuille flow at leading order; furthermore, viscous dissipation does not appear at leading order, so without pressure work there is no change to the temperature.

To solve (32) it is convenient to let

$$
N=4 p^{4}
$$


note that $p$ is proportional to the duct half-width $L$. We then find

$$
w_{1}=-\frac{\sinh p(1+y) \sin p(1-y)+\sinh p(1-y) \sin p(1+y)}{p(\sinh 2 p+\sin 2 p)},
$$

with

$$
\begin{gathered}
\theta_{1}-\theta_{1 w}=\frac{2 p}{\sinh 2 p+\sin 2 p}[\cosh 2 p+\cos 2 p-\cosh p(1+y) \cos p(1-y) \\
-\cosh p(1-y) \cos p(1+y)]
\end{gathered}
$$

in which the temperature profile is given relative to the fixed wall temperature, indicated by subscript $w$.

The solution (37) and (38) is mathematically similar to that obtained by Tao [14] for the case where a uniform streamwise temperature gradient is imposed. Without boundary constraints, pressure work would produce a vertical (adiabatic) lapse rate in the temperature: thus Tao's analysis with an imposed vertical temperature gradient but no pressure work replicates our analysis which includes pressure work but specifies vertically uniform temperature. In the limit $p \rightarrow 0,(37)$ approaches the Poiseuille flow solution (35); however, the case where the pressure work parameter $p$ is larger is of more interest. According to (37) and (38), the vertical velocity and the temperature oscillate in the cross-stream direction, with the amplitude of oscillations decreasing from the walls towards the mid-plane of the duct. Such oscillations in vertical flows with significant pressure work have previously been discussed by Kuiken [3]: by cooling a rising fluid or warming a descending fluid, pressure work produces a buoyancy force which always opposes the direction of motion. Equation (32) is a leading-order energy balance between conduction and pressure work. The applied forcing, appearing through the shear-stress boundary condition at the walls, drives a flow which produces a temperature change through pressure work. This temperature change is conducted inwards from the wall, and its associated buoyancy force will at some location drive a flow opposed to that adjacent to the wall. That flow will then induce an opposite temperature change due to pressure work, and further inward conduction of heat will lead to further flow reversals by the same mechanism. Flow and temperature profiles at $O(\lambda)$ are shown by long-dashed curves in Figure 1: oscillations are not apparent with $N=4$ because their wavelength is too great, while at $N=4 \times 10^{4}$ the exponential decay of their amplitude away from the wall is clear.

An alternative interpretation of the large- $N$ flow profile is that the leadingorder solution of (17) as $N \rightarrow \infty$ is $w \sim 0$, but this does not satisfy the shear-stress boundary condition so there is a boundary layer of thickness $\delta \sim$ $N^{-1 / 4}$ adjacent to the wall. The velocity magnitude in the boundary layer is controlled by the wall shear stress, so $w \sim \lambda N^{-1 / 4}$. Viscous dissipation is $(\mathrm{d} w / \mathrm{d} y)^{2} \sim \lambda^{2}$ in the boundary layer, so can only be of similar magnitude to pressure work if $\lambda \sim N^{3 / 4}$ : since $\lambda$ and $N$ are both proportional to $L^{4}$, the pressure gradient required to produce significant dissipative heating in a wide 
duct is inversely proportional to duct width. For smaller values of $\lambda$ with large $N$, the full solution of (17) differs little from that given by the leading-order energy balance between conduction and pressure work in (32), as seen in the right-hand panels of Figure 1 in which higher-order terms make no noticeable difference to the solution. Since the conduction term in (17) can be written as $\mathrm{d}^{2} \theta / \mathrm{d} y^{2}$, a scaling for the temperature difference across the boundary layer is $\theta-\theta_{w} \sim N w \delta^{2} \sim \lambda N^{1 / 4}$, consistent with (38) which yields $\theta_{1}-\theta_{1 w} \approx 2 p$ for $y$ not close to \pm 1 , and with the value of 200 shown in Figure 1 with $\lambda=10$ and $p=10$.

At $O\left(\lambda^{2}\right),(18)$ yields

$$
\frac{\mathrm{d}^{4} w_{2}}{\mathrm{~d} y^{4}}=\left(\frac{\mathrm{d} w_{1}}{\mathrm{~d} y}\right)^{2}
$$

while we obtain

$$
\frac{\mathrm{d}^{4} w_{2}}{\mathrm{~d} y^{4}}+N w_{2}=\left(\frac{\mathrm{d} w_{1}}{\mathrm{~d} y}\right)^{2}
$$

from (17). The boundary conditions are

$$
w_{2}^{\prime}(0)=0, \quad w_{2}^{\prime \prime \prime}(0)=0, \quad w_{2}(1)=0, \quad w_{2}^{\prime}(1)=0,
$$

and the temperature perturbation at $O\left(\lambda^{2}\right)$ is found from

$$
\theta_{2}=-\frac{\mathrm{d}^{2} w_{2}}{\mathrm{~d} y^{2}}
$$

Inserting the respective solutions for $w_{1}$ into the right-hand sides of (39) and (40), and solving with the boundary conditions (41), we obtain

$$
w_{2}=\frac{1}{360}\left(1-y^{2}\right)^{2}\left(2+y^{2}\right), \quad \theta_{2}-\theta_{2 w}=\frac{1}{12}\left(1-y^{2}\right)\left(1+y^{2}\right)
$$

without pressure work, and

$$
\begin{aligned}
w_{2}=\frac{1}{120 p^{4}(\sinh 2 p+\sin 2 p)^{2}} \times \\
\quad[30 \sinh 2 p \sin 2 p-2 \sinh 2 p y \sin 2 p y \\
\quad+6(\cosh 2 p+\cos 2 p)(\cosh 2 p y-\cos 2 p y) \\
+\sinh 2 p(1+y) \sin 2 p(1-y)+\sinh 2 p(1-y) \sin 2 p(1+y) \\
+8(\cosh 2 p+\cos 2 p)(\sinh p(1+y) \sin p(1-y)+\sinh p(1-y) \sin p(1+y)) \\
-\left(6(\sinh 2 p+\sin 2 p)+\frac{16 \sinh 2 p \sin 2 p}{\sinh 2 p+\sin 2 p}\right) \times \\
\quad(\cosh p(1+y) \sin p(1-y)+\cosh p(1-y) \sin p(1+y) \\
\quad+\sinh p(1+y) \cos p(1-y)+\sinh p(1-y) \cos p(1+y))],
\end{aligned}
$$




$$
\begin{aligned}
\theta_{2}-\theta_{2 w}= & \frac{1}{30 p^{2}(\sinh 2 p+\sin 2 p)^{3}} \times \\
& {\left[\begin{array}{c}
(\sinh 2 p-\sin 2 p)^{3}+12 \sinh 2 p \sin 2 p(\sinh 2 p-\sin 2 p) \\
+
\end{array}\right.} \\
& +(\sinh 2 p+\sin 2 p) \cosh 2 p y \cos 2 p y \\
+ & (\cosh 2 p+\cos 2 p)(\sinh 2 p+\sin 2 p)(-6(\cosh 2 p y+\cos 2 p y) \\
& +4(\cosh p(1+y) \cos p(1-y)+\cosh p(1-y) \cos p(1+y))) \\
+ & +(3(\sinh 2 p+\sinh 2 p)(\cosh 2 p(1+y) \cos 2 p(1-y)+\cosh 2 p(1-y) \cos 2 p(1+y)) \\
& \quad(\cosh p(1+y) \sin p(1-y)-\sinh p(1+y) \cos p(1-y) \\
& +(\cosh p(1-y) \sin p(1+y)-\sinh p(1-y) \cos p(1+y))]
\end{aligned}
$$

in the formulation including pressure work. The large numerical coefficients in the denominators of $(43)$ - (45) suggest that the expansions may provide good approximations when $\lambda$ is not small; although convergence has not been analysed, we have obtained the third term in the case without pressure work,

$$
\begin{gathered}
w_{3}=-\frac{1}{151200}\left(1-y^{2}\right)^{2}\left(3+y^{2}\right)\left(8+y^{2}-y^{4}\right), \\
\theta_{3}-\theta_{3 w}=-\frac{1}{5040}\left(1-y^{2}\right)\left(1+y^{2}\right)\left(11-3 y^{4}\right)
\end{gathered}
$$

(i.e. yielding $\left|w_{3}\right| \leq 1 / 6300$ for $|y| \leq 1$ ), which reinforces this suggestion. The expansions to $O\left(\lambda^{2}\right)$ are shown by solid curves in Figure 1, and their closeness to the numerical solutions of (18) and (17) with $\lambda=10$ (shown by short-dashed curves) confirms that the perturbation solutions yield rather good approximations even with this fairly large value of $\lambda$. We have already noted that $\lambda=10$ with $N=4$ implies a dynamic pressure gradient not very small compared with hydrostatic; an a posteriori check on whether the Boussinesq approximation is valid is that temperature variations should be small compared with the absolute temperature, which according to (27) means that the variation in $\theta$ across the duct should be small compared with $N$. From Figure 1 this is satisfied with $N=4 \times 10^{4}$ but certainly not in the case $N=4$; with pressure work excluded, $N$ is arbitrary so the issue does not arise except that an acceptably large $N$ may imply a large duct width. Nevertheless, whereas a smaller value of $\lambda$ would be more physically realistic when $N$ is also of moderate size, we show solutions with $\lambda=10$ to give clearer illustrations of the effects of various terms in the perturbation expansions.

Since viscous dissipation first appears in the perturbation equations at $O\left(\lambda^{2}\right)$, the difference between the long-dashed curves $(O(\lambda)$ expansions) and the solid curves $\left(O\left(\lambda^{2}\right)\right.$ expansions) includes the lowest-order effect of viscous dissipation. This difference appears much more significant in the temperature 

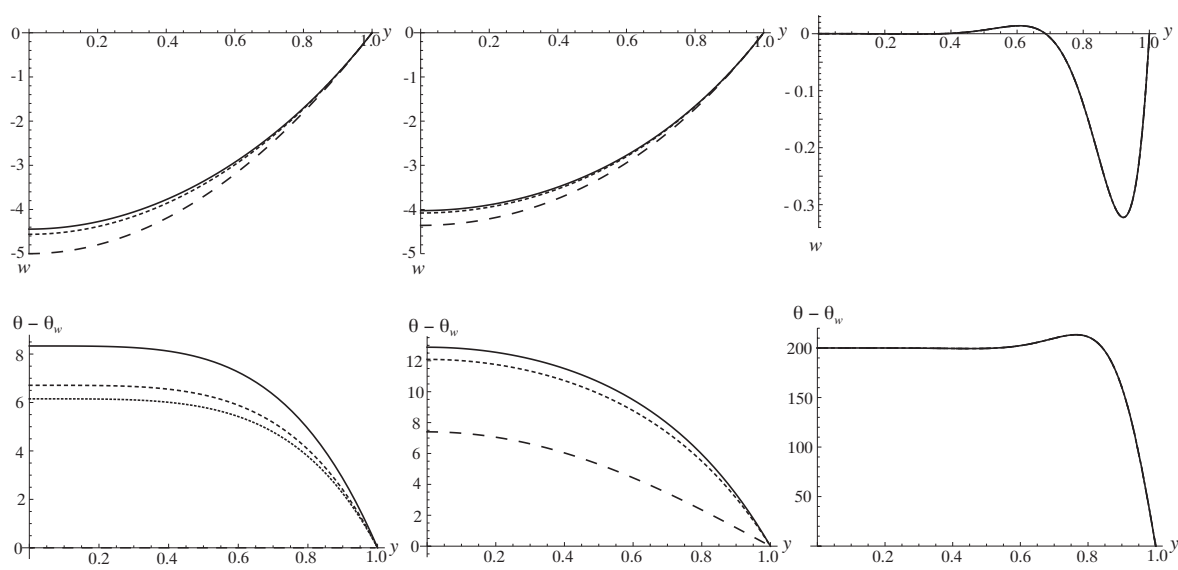

Fig. 1 Velocity profiles (upper row) and temperature profiles relative to wall temperature $\theta_{w}$ (lower row), between axis of symmetry and wall. Dimensionless pressure gradient $\lambda=10$, and wall temperatures are held equal. Left: ignoring pressure work. Centre: with $N=4$. Right: with $N=4 \times 10^{4}$. Short-dashed curves: numerical solutions. Long-dashed curves: expansions to $O(\lambda)$. Solid curves: expansions to $O\left(\lambda^{2}\right)$. Lower left plot also contains dotted curve, for expansion to $O\left(\lambda^{3}\right)$. In the right-hand plots the three curves are all present, but indistinguishable.

profiles than the velocity profiles. The velocity profile is primarily driven by the pressure gradient, and then modified due to pressure work and viscous dissipation, whereas temperature perturbations are entirely due to the latter two effects. With $N=4$, heating at $O\left(\lambda^{2}\right)$ results in an approximately $10 \%$ reduction in downward velocity, whereas the temperature rise at $O(\lambda)$ only accounts for around $60 \%$ of the total temperature rise as given by the numerical solution, which the $O\left(\lambda^{2}\right)$ expansion slightly overshoots. If pressure work is excluded so that dissipation is the only heating mechanism, the $O\left(\lambda^{2}\right)$ temperature rise overshoots the true value by around $25 \%$, but adding the $O\left(\lambda^{3}\right)$ term produces a better approximation. Note that the flatness of the temperature profile away from the wall in this case is for a different reason than in the case $N=4 \times 10^{4}$. Viscous dissipation depends on velocity gradients whereas pressure work is proportional to velocity; when pressure work is excluded it is the small velocity gradients near the duct mid-plane that give rise to a flat temperature profile, whereas when pressure work is dominant it is the small velocities near the mid-plane that yield a similar result.

Viscous dissipation is the same for upward and downward flow, whereas pressure work changes sign with the velocity. Hence we do not expect results with negative $\lambda$ to mirror those with positive $\lambda$. Results with $\lambda=-10$ are shown in Figure 2. For the case $N=4 \times 10^{4}$, where pressure work is dominant, the velocity and temperature profiles are simply inverted versions of the profiles with $\lambda=10$, but this is not true in the other cases. Where pressure work is excluded, heating by viscous dissipation increases the magnitude of upward velocity (with negative $\lambda$ ) whereas it works against downward flow. The 

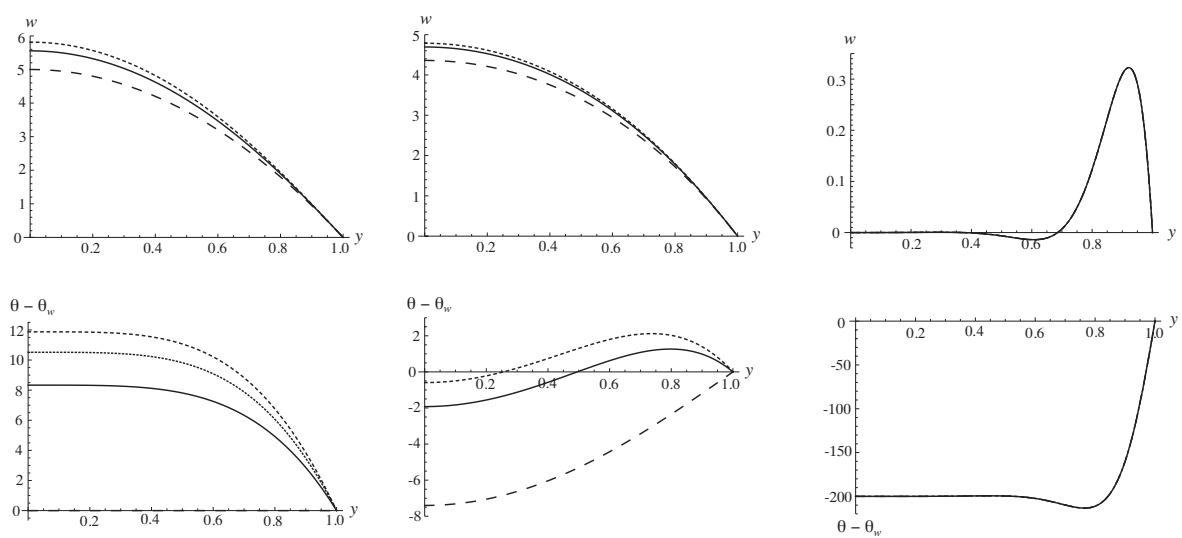

Fig. 2 Velocity and temperature profiles as in Figure 1, but with $\lambda=-10$.

temperature rise at $O\left(\lambda^{2}\right)$ is due to the leading-order Poiseuille flow and so is the same for flow in either direction, but at higher order it becomes greater in upward flow and less in downward flow as dissipative heating is affected by perturbations to the Poiseuille flow, with velocity gradients roughly in proportion to velocities. In the intermediate case with $N=4$, effects on the velocity profile are similar: a Poiseuille profile, reduced in magnitude at leading order due to pressure work, is then enhanced in upward flow but further reduced in downward flow due to dissipative heating at $O\left(\lambda^{2}\right)$. But the temperature profiles in this case are more striking: at leading order the temperature rise in downward flow is mirrored by a temperature fall in upward flow, but viscous dissipation then reverses the latter temperature change in the part of the domain with the greatest velocity gradients. However, as has already been remarked, the value $|\lambda|=10$ has been chosen to make higher-order effects clear on the plots but with $N=4$ the results are not physically realistic; solutions with $\lambda=-1$ (not shown) have dissipation at $O\left(\lambda^{2}\right)$ making a more modest reduction in the pressure-work cooling. Possibly the most important point is that in upward flow a formulation in which pressure work is excluded leads to temperature changes of opposite sign to those found when pressure work is included.

\subsection{Forced convection with insulated walls}

If the walls are insulated while there is heating or cooling by pressure work and/or heating by viscous dissipation, there will be an increase or decrease in the heat contained within the duct. This heat excess/deficit may remain in situ, resulting in a variation of temperature with time, or it may be convected downstream, resulting in streamwise variation of temperature. In general there may be a combination of temporal and streamwise temperature variation: both are accounted for in equation (15). If entry and exit conditions were given, the 
streamwise temperature gradient would be constrained; this is not done in our formulation of fully-developed flow, so there is a degree of arbitrariness. We are free to specify either the streamwise or temporal temperature variation to be zero, and we shall solve equations for both cases, denoted respectively "temporal heating" and "streamwise heating" (although there may be cooling rather than heating if pressure work is dominant in upward flow).

\subsubsection{Temporal heating}

We set $\gamma=0$ in (20) and (21). As well as expanding the velocity and temperature according to (29) and (30), the heating rate parameter $\eta$ must be expanded:

$$
\eta=\eta_{0}+\lambda \eta_{1}+\lambda^{2} \eta_{2}+\ldots
$$

However, before presenting the solutions at each order in $\lambda$, we derive some scalings for $\eta$, assuming in all cases that $\lambda$ is $O(1)$ or smaller. If pressure work is excluded, (21) with the boundary conditions (22) and with $\gamma=0$ yields

$$
\eta=\int_{0}^{1}\left(\frac{\mathrm{d} w}{\mathrm{~d} y}\right)^{2} \mathrm{~d} y .
$$

The shear-stress boundary condition $w^{\prime}(1)=\lambda$ then implies

$$
\eta \sim \lambda^{2}
$$

If pressure work is included and is a more significant source of heating/cooling than viscous dissipation, but not a dominant factor in the dynamics, then (20) yields $\eta \sim-N w$ where the velocity is still determined principally by the dynamic pressure gradient, i.e from the boundary condition $w^{\prime}(1)=\lambda$. So $|w| \sim \lambda$, leading to

$$
|\eta| \sim \lambda N
$$

Finally, if the pressure work parameter $N$ is large, the velocity will be determined by the balance between heating rate and pressure work in the energy equation (20): $w \sim-\eta / N$. However, this uniform, non-zero velocity is inconsistent with the no-slip boundary condition, so there must be a boundary layer in which the conduction term $\mathrm{d}^{4} w / \mathrm{d} y^{4}$ in (20) is of similar magnitude to the pressure work term $N w$, a balance which yields a boundary layer thickness $\sim N^{-1 / 4}$. The velocity gradient in this boundary layer is determined by the wall shear stress, so the velocity outside the boundary layer is $|w| \sim \lambda N^{-1 / 4}$. Hence the heating rate is

$$
|\eta| \sim N w \sim \lambda N^{3 / 4}
$$

Proceeding to the expansion in powers of $\lambda$, at $O(1)$ we again have only the trivial solution, $w_{0} \equiv 1$, while at $O(\lambda)$ we have

$$
\frac{\mathrm{d}^{4} w_{1}}{\mathrm{~d} y^{4}}=-\eta_{1}
$$


in the formulation without pressure work, or

$$
\frac{\mathrm{d}^{4} w_{1}}{\mathrm{~d} y^{4}}+N w_{1}=-\eta_{1}
$$

when pressure work is included. The boundary conditions in both cases are

$$
w_{1}^{\prime}(0)=0, \quad w_{1}^{\prime \prime \prime}(0)=0, \quad w_{1}(1)=0, \quad w_{1}^{\prime}(1)=1, \quad w_{1}^{\prime \prime \prime}(1)=0 ;
$$

the problem is well-posed with five boundary conditions on a fourth-order ODE containing one parameter $\eta_{1}$ to be determined.

Without pressure work there is no heating at $O(\lambda)$, so we have the same Poiseuille flow (35) as with constant-temperature walls, with $\eta_{1}=0$. However, when pressure work is included,

$$
\eta_{1}=2 p^{3} \frac{\sinh 2 p-\sin 2 p}{\cosh 2 p-\cos 2 p},
$$

where

$$
p=\frac{N^{1 / 4}}{\sqrt{2}}
$$

as before. Clearly $\eta_{1}$ is positive for positive $N$, so the leading-order dimensionless rate of temperature change $\lambda \eta_{1}$ has the same sign as $\lambda$, i.e. cooling for upward flow and warming for downward flow as expected. For small or large values of the pressure work parameter $N$, we have

$$
\eta_{1} \sim \frac{1}{3} N \text { as } N \rightarrow 0, \quad \eta_{1} \sim \frac{1}{\sqrt{2}} N^{3 / 4} \text { as } N \rightarrow \infty,
$$

consistent with the scalings derived above. The $O(\lambda)$ velocity and temperature perturbation are

$$
\begin{gathered}
w_{1}=\frac{1}{2 p(\cosh 2 p-\cos 2 p)}[-\sinh 2 p+\sin 2 p \\
+\sinh p(1+y) \cos p(1-y)+\sinh p(1-y) \cos p(1+y) \\
-\cosh p(1+y) \sin p(1-y)-\cosh p(1-y) \sin p(1+y)], \quad \text { (59) } \\
\theta_{1}=1-\frac{p}{(\cosh 2 p-\cos 2 p)}[\cosh p(1+y) \sin p(1-y)+\cosh p(1-y) \sin p(1+y) \\
+\sinh p(1+y) \cos p(1-y)+\sinh p(1-y) \cos p(1+y)], \quad(60)
\end{gathered}
$$

shown by long-dashed curves in Figure 3, which is plotted for the case of downward flow (positive $\lambda$ ).

Temperature changes at $O(\lambda)$ occur only if pressure work is included. With $N=4$, the warming is greatest at the mid-plane of the duct where the downward velocity is greatest, and there is only a small perturbation to the Poiseuille flow profile, with downward velocities being reduced slightly due to the warming. For large $N$, when pressure work is a dominant effect, the velocity away from the walls is approximately $-\lambda \eta_{1} / N \sim-\lambda N^{-1 / 4} / \sqrt{2}=-0.5$ 

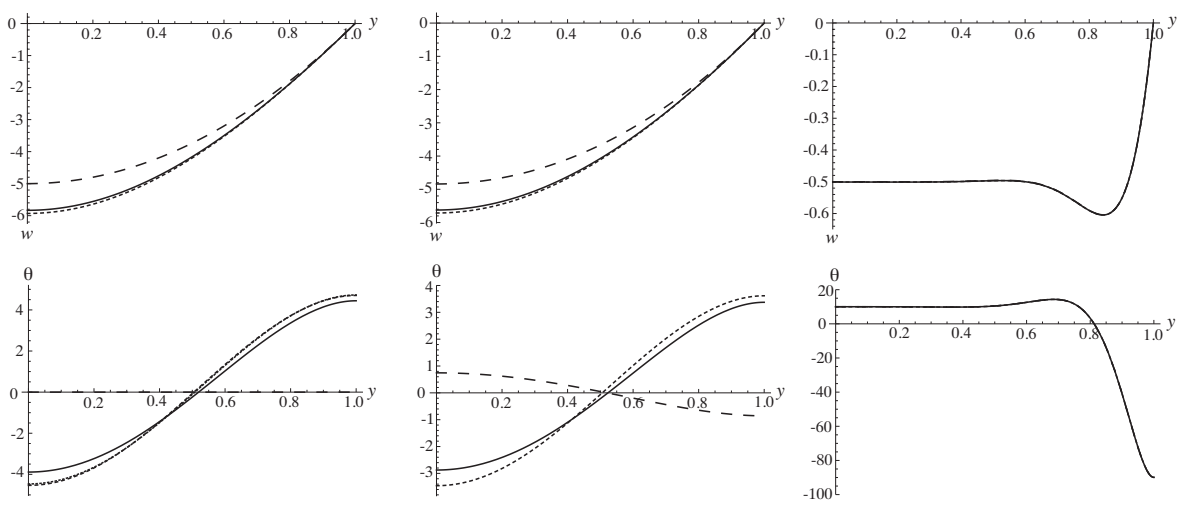

Fig. 3 Velocity and temperature profiles (relative to cross-section mean) for forced convection with insulated walls and temporal heating. Dimensionless pressure gradient $\lambda=10$. Other details as in Figure 1.

with $\lambda=10$ and $N=4 \times 10^{4}$. This approximately uniform flow produces an approximately uniform temperature perturbation $\theta \sim \lambda$ (from (16)) away from the duct walls, but $\theta \sim \lambda(1-p)$ at the walls according to (60). As in the case of fixed wall temperature, the velocity and temperature profiles are in principle oscillatory, but with small $N$ the wavelength of oscillations is greater than the duct width whereas with large $N$ they decay exponentially away from the walls.

At $O\left(\lambda^{2}\right),(20)$ and (21) yield respectively

$$
\frac{\mathrm{d}^{4} w_{2}}{\mathrm{~d} y^{4}}=\left(\frac{\mathrm{d} w_{1}}{\mathrm{~d} y}\right)^{2}-\eta_{2},
$$

without pressure work, and

$$
\frac{\mathrm{d}^{4} w_{2}}{\mathrm{~d} y^{4}}+N w_{2}=\left(\frac{\mathrm{d} w_{1}}{\mathrm{~d} y}\right)^{2}-\eta_{2}
$$

when pressure work is included. Boundary conditions are

$$
w_{2}^{\prime}(0)=0, \quad w_{2}^{\prime \prime \prime}(0)=0, \quad w_{2}(1)=0, \quad w_{2}^{\prime}(1)=0, \quad w_{2}^{\prime \prime \prime}(1)=0 .
$$

The solutions are

$$
w_{2}=-\frac{1}{360}\left(1-y^{2}\right)^{2}\left(3-y^{2}\right), \quad \theta_{2}=\frac{2}{45}-\frac{1}{12}\left(1-y^{2}\right)^{2}
$$

without pressure work, and

$$
\begin{aligned}
w_{2}= & \frac{1}{240 p^{4}(\cosh 2 p-\cos 2 p)^{2}} \times \\
& {\left[-20\left(\sinh ^{2} 2 p-\sin ^{2} 2 p\right)+10(\cosh 2 p \cos 2 p-1)\right.}
\end{aligned}
$$




$$
\begin{aligned}
& +6(\cosh 2 p-\cos 2 p)(\cosh 2 p y-\cos 2 p y)+2 \cosh 2 p y \cos 2 p y \\
& -\cosh 2 p(1+y) \cos 2 p(1-y)-\cosh 2 p(1-y) \cos 2 p(1+y) \\
& +16(\cosh 2 p+\cos 2 p)(\cosh p(1+y) \cos p(1-y)+\cosh p(1-y) \cos p(1+y)) \\
& +24(\cosh 2 p-\cos 2 p)(\sinh p(1+y) \sin p(1-y)+\sinh p(1-y) \sin p(1+y)) \\
& -32(\cosh p(1+y) \cos p(1+y)+\cosh p(1-y) \cos p(1-y))]
\end{aligned}
$$

$$
\begin{aligned}
& \theta_{2}=\frac{1}{30 p^{2}(\cosh 2 p-\cos 2 p)^{2}} \times \\
& \quad-3(\cosh 2 p-\cos 2 p)(\cosh 2 p y+\cos 2 p y)+2 \sinh 2 p y \sin 2 p y \\
&+\sinh 2 p(1+y) \sin 2 p(1-y)+\sinh 2 p(1-y) \sin 2 p(1+y) \\
&-4(\cosh 2 p+\cos 2 p)(\sinh p(1+y) \sin p(1-y)+\sinh p(1-y) \sin p(1+y)) \\
&+6(\cosh 2 p-\cos 2 p)(\cosh p(1+y) \cos p(1-y)+\cosh p(1-y) \cos p(1+y) \\
&-8(\sinh p(1+y) \sin p(1+y)+\sinh p(1-y) \sin p(1-y))],
\end{aligned}
$$

when pressure work is included. The heating rate is

$$
\eta_{2}=\frac{1}{3}
$$

in both cases: this is the value of heating rate produced by the Poiseuille flow profile found at $O(\lambda)$ without pressure work, and although that profile is perturbed when pressure work is present, the resulting change in heat generation by viscous dissipation appears to be exactly compensated by heat generated by pressure work at $O\left(\lambda^{2}\right)$.

Temerature profiles resulting from viscous dissipation at $O\left(\lambda^{2}\right)$ in Figure 3 are very different from those occurring with fixed wall temperatures (Figure 1). In both cases, heat is generated mainly near the duct walls, where the velocity gradient is greatest: with fixed-temperature walls, heat can be conducted out through those walls as well as in towards the mid-plane of the duct, so the greatest temperatures are found near the mid-plane; but when the walls are insulated, the heat cannot be conducted out so the highest temperatures are found where the heat is being generated. The relatively cooler temperatures near the mid-plane in the latter case enhance the downward flow in this region. These effects are seen in Figure 3 both in the case where pressure work is excluded and where $N=4$; in the latter case, viscous dissipation apparently reverses the temperature trends produced by pressure work at $O(\lambda)$, but this is an artefact of the unrealistically large value of $\lambda$ used in the plots; with $\lambda=1$, the temperature profile at $O\left(\lambda^{2}\right)$ (and in the numerical solution) is of similar shape to that at $O(\lambda)$, but reduced in magnitude. For $N=4 \times 10^{4}$, viscous dissipation makes negligible change to the velocity and temperature profiles which are controlled by pressure work. 
Given that $\lambda=10$ is a rather large value of the "small" parameter in the perturbation expansion, there is surprisingly good agreement between values of $\eta$ obtained in the numerical solutions and those found from the first few terms of the expansion. This is particularly true in the case where pressure work is dominant: with $N=4 \times 10^{4}, p=10$, the numerical solution yields $\eta=20,033.32$, agreeing to six significant figures with

$$
\eta=2 p^{3} \frac{\sinh 2 p-\sin 2 p}{\cosh 2 p-\cos 2 p} \lambda+\frac{1}{3} \lambda^{2}
$$

(and to three significant figures with the first term in the expansion). With $N=4, p=1$, the numerical solution gives $\eta=55.8858$ whereas (68) gives $\eta=46.3412$, with a substantially greater contribution from the second term (due to dissipative heating) than the first term (pressure work). If pressure work is excluded, the numerical solution gives $\eta=43.4787$; at $O\left(\lambda^{2}\right)$ in this case, we have $\eta \sim \lambda^{2} / 3=33 \frac{1}{3}$, but a further term in this expansion has been calculated,

$$
\eta=\frac{1}{3} \lambda^{2}+\frac{16}{1890} \lambda^{3}
$$

yielding a value of 41.7989 as the $O\left(\lambda^{3}\right)$ approximation to $\eta$.

We may ask whether these values of dimensionless heating rate $\eta$ are physically reasonable. For this purpose we note that the conduction time scale over a distance $L$ is

$$
t_{c}=\frac{L^{2}}{k / c \rho_{m}}
$$

and that the fractional temperature change over this time scale is

$$
\frac{t_{c}}{T_{m}} \frac{\mathrm{d} T}{\mathrm{~d} t}=\frac{\eta}{N}
$$

(from (13) and (14)). The duct width $L$ is tied to the value of $N$ for any given fluid, and the values of $t_{c}$ with $N=4$ are $9.9 \times 10^{3} \mathrm{~s}$ for water and $1.4 \times 10^{5} \mathrm{~s}$ for glycerine, both at $20^{\circ} \mathrm{C}$. We have found $\eta / N \approx 14$ with $\lambda=10$ and $N=4$, so the temperature change in water would only remain small compared to the absolute temperature for a matter of minutes - but as we have already observed, $\lambda=10$ does yield somewhat unrealistic results with $N=4$, and the rate of temperature change would be more reasonable with a smaller value of $\lambda$. With $N=4 \times 10^{4}$ the time scale $t_{c}$ is 100 times greater than with $N=4$, and we have found $\eta / N \approx 2$, so the heating rate is more realsitic although this value of $N$ does imply a rather large duct.

Differences in velocity and temperature profiles between upwardly forced flow (negative $\lambda$ ) and downward flow have been discussed in Section 3.1; the same principles apply in the case of insulated walls, so we do not show results computed with negative $\lambda$ here. 


\subsubsection{Streamwise heating}

We set $\eta=0$ in (20) and (21), and observe that the equation including pressure work is now of the same form as that without pressure work, but with $\gamma$ replaced by $N+\gamma$. Thus the velocity and temperature profiles will be the same in both cases, and the only change if pressure work is included will be that the dimensionless streamwise temperature gradient will differ by an amount $N$. From (20), we obtain

$$
\gamma=-N+\int_{0}^{1}\left(\frac{\mathrm{d} w}{\mathrm{~d} y}\right)^{2} \mathrm{~d} y / \int_{0}^{1} w \mathrm{~d} y
$$

in which the contribution from viscous dissipation (= dissipation rate / volume flux) is always $O(\lambda)$, while the contribution from pressure work is simply the adiabatic lapse rate, as can be seen by using (13) and (14) to write the streamwise (vertical) temperature gradient in dimensioned variables:

$$
\frac{\mathrm{d} T}{\mathrm{~d} Z}=\frac{\Gamma}{N} \gamma
$$

where $\Gamma$ is the adiabatic lapse rate,

$$
\Gamma=\frac{g \beta T_{m}}{c}
$$

(in which we have assumed that the appropriate specific heat $c$ does not differ appreciably from $c_{p}$, as should be the case in liquids). Values of $\Gamma$ are $1.4 \times$ $10^{-4}{ }^{\circ} \mathrm{C} . \mathrm{m}^{-1}$ for water and $5.5 \times 10^{-4}{ }^{\circ} \mathrm{C} . \mathrm{m}^{-1}$ for glycerine, both at $20^{\circ} \mathrm{C}$. If $N \gg \lambda$, so that pressure work is the main source of heating/cooling, the total streamwise (i.e. vertical) temperature gradient will be given approximately by the adiabatic lapse rate. In other cases, assuming always that $\lambda \sim O(1)$, the streamwise temperature gradient could only be unreasonably large if $N$ is rather small, i.e. for a narrow duct.

The reason that pressure work does not affect the dynamics is that the streamwise (vertical) temperature variation is not constrained by boundary conditions in the present case. We could remove pressure work from the equations entirely by replacing the temperature with a potential temperature, as is commonly done in meteorology; but even without doing that, we only need to solve equation (21), in which pressure work is excluded. Expanding the velocity and temperature according to (29) and (30), and the streamwise temperature gradient as

$$
\gamma=\gamma_{0}+\lambda \gamma_{1}+\lambda^{2} \gamma_{2}+\ldots
$$

we again find $w_{0} \equiv 0$ at $O(1)$. Then at $O(\lambda)(21)$ yields

$$
\frac{\mathrm{d}^{4} w_{1}}{\mathrm{~d} y^{4}}+\gamma_{0} w_{1}=0
$$


with the boundary conditions (55). The solution at $O(\lambda)$ is again Poiseuille flow with no heating:

$$
w_{1}=-\frac{1}{2}\left(1-y^{2}\right), \quad \theta_{1}=0, \quad \gamma_{0}=0 .
$$

Including pressure work would simply set $\gamma_{0}=-N$ at this stage, and there would be no change to subsequent calculations. At $O\left(\lambda^{2}\right)$ we have

$$
\frac{\mathrm{d}^{4} w_{2}}{\mathrm{~d} y^{4}}=\left(\frac{\mathrm{d} w_{1}}{\mathrm{~d} y}\right)^{2}-\gamma_{1} w_{1},
$$

with boundary conditions (63). The solution is

$$
w_{2}=-\frac{1}{240}\left(1-y^{2}\right)^{2}\left(3-y^{2}\right), \quad \theta_{2}=\frac{1}{15}-\frac{1}{8}\left(1-y^{2}\right)^{2}
$$

with $\gamma_{1}=-1$. The velocity and temperature perturbations at this order are simply $3 / 2$ times those in the case of temporal heating. The leading-order dimensionless temperature gradient is $\lambda \gamma_{1}=-\lambda$, which is a temperature increase in the direction of flow, as expected when heat is generated by viscous dissipation. To obtain $\gamma_{2}$ we need the solution at $O\left(\lambda^{3}\right)$ : we have

$$
\frac{\mathrm{d}^{4} w_{3}}{\mathrm{~d} y^{4}}=2 \frac{\mathrm{d} w_{1}}{\mathrm{~d} y} \frac{\mathrm{d} w_{2}}{\mathrm{~d} y}-\gamma_{1} w_{2}-\gamma_{2} w_{1}=0
$$

with boundary conditions on $w_{3}$ of the same form as those on $w_{2}$, yielding

$$
\gamma_{2}=-\frac{2}{105}
$$

with

$$
\begin{gathered}
w_{3}=-\frac{1}{1209600}\left(1-y^{2}\right)^{2}\left(419-291 y^{2}+109 y^{4}-13 y^{6}\right), \\
\theta_{3}=-\frac{56}{32175}-\frac{1}{11440}\left(1-y^{2}\right)^{3}\left(45-13 y^{2}\right) .
\end{gathered}
$$

Velocity and temperature profiles are rather similar to those found with temporal heating in the absence of pressure work, so by way of contrast to Figure 3, we show profiles for upward flow (negative $\lambda$ ) in Figure 4. Dissipative heating is greatest near the wall, where the velocity gradient has largest magnitude. The relatively cooler fluid near the mid-plane experiences a downward buoyancy force, reducing the velocity of an upward flow at $O\left(\lambda^{2}\right)$ (Figure 4) or increasing a downward velocity (Figure 3 ). This in turn reduces the magnitude of dissipative heating in upward flow, but increases it in downward flow; the consequent increase or decrease of the cross-stream temperature contrast at $O\left(\lambda^{3}\right)$ changes the buoyancy force and hence affects the velocity profile at $O\left(\lambda^{3}\right)$. 

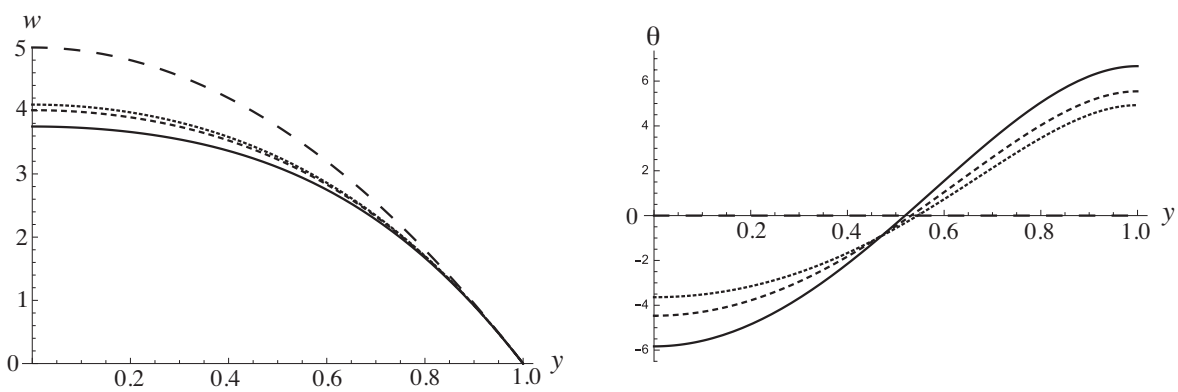

Fig. 4 Velocity and temperature profiles (relative to cross-section mean) for forced convection with insulated walls and streamwise heating. Dimensionless pressure gradient $\lambda=-10$. Expansions to $O(\lambda)$ (long-dashed curves), $O\left(\lambda^{2}\right)$ (solid curves) and $O\left(\lambda^{3}\right)$ (dotted curves), together with numerical solution (short-dashed curves).

3.3 Free convection with fixed wall temperatures

To solve (17) and (18) with the boundary conditions (24), we expand in powers of the dimensionless imposed temperature difference $\Theta$ :

$$
\begin{gathered}
w=w_{0}+\Theta w_{1}+\Theta^{2} w_{2}+\ldots, \\
\theta=\theta_{0}+\Theta \theta_{1}+\Theta^{2} \theta_{2}+\ldots
\end{gathered}
$$

As with forced convection, we obtain $w_{0} \equiv 0$. Next, equations (31) and (32) apply at $O(\Theta)$, but with boundary conditions

$$
w_{1}(-1)=0, \quad w_{1}(1)=0, \quad w_{1}^{\prime}(1)-w_{1}^{\prime}(-1)=0, \quad w_{1}^{\prime \prime}(1)-w_{1}^{\prime \prime}(-1)=-1 .
$$

Without pressure work, we find the velocity and temperature profiles associated with simple conduction of heat from the hotter to the cooler wall:

$$
w_{1}=\frac{1}{12} y\left(1-y^{2}\right), \quad \theta_{1}-\theta_{1 M}=\frac{1}{2} y .
$$

If pressure work is included, the solution of (32) is

$$
w_{1}=\frac{\sinh p(1+y) \sin p(1-y)-\sinh p(1-y) \sin p(1+y)}{4 p^{2}(\cosh 2 p-\cos 2 p)}
$$

with

$$
\theta_{1}-\theta_{1 M}=\frac{\cosh p(1+y) \cos p(1-y)-\cosh p(1-y) \cos p(1+y)}{2(\cosh 2 p-\cos 2 p)} .
$$

With no viscous dissipation at $O(\Theta)$, these profiles are antisymmetric, and are shown by long-dashed curves in Figure 5, in which we display profiles for $N=400$ rather than $N=4$ as was done for forced convection, since in the present case the profiles with $N=4$ differ little from those in which pressure 
work is excluded. For these antisymmetric profiles, $\theta_{1 M}=0$, i.e. at $O(\Theta)$ the mean of the wall temperatures is equal to the cross-section mean.

Most of the comments in Section 3.1 relating to the effects of pressure work still apply here; indeed, in the case $N=4 \times 10^{4}$ where pressure work dominates, the velocity profiles in Figures 2 and 5 are very similar. In both cases the buoyancy force resulting from the cooling/heating due to pressure work opposes the primary flow; if $N$ is large, this results in the fluid being almost stationary except near the walls. The difference between the two cases is that here it is a temperature boundary condition which cannot be satisfied by static fluid, whereas in forced convection it was a shear-stress boundary condition. The boundary layer thickness is still $\delta \sim N^{-1 / 4}$, but the velocity in the boundary layer is now given by (23) as $w \sim \Theta \delta^{2} \sim \Theta N^{-1 / 2}$. Viscous dissipation in the boundary layer is then $(\mathrm{d} w / \mathrm{d} y)^{2} \sim \Theta^{2} N^{-1 / 2}$, and so is only of similar magnitude to pressure work if $\Theta \sim N$, which would certainly invalidate the Boussinesq approximation since it means that the temperature difference between the walls is of similar magnitude to the absolute mean temperature.

At $O\left(\Theta^{2}\right)$, equations (39) and (40) apply, with boundary conditions

$$
w_{2}(-1)=0, \quad w_{2}(1)=0, \quad w_{2}^{\prime}(1)-w_{2}^{\prime}(-1)=0, \quad w_{2}^{\prime \prime}(1)-w_{2}^{\prime \prime}(-1)=0 .
$$

The solution without pressure work is

$w_{2}=\frac{1}{241920}\left(1-y^{2}\right)^{2}\left(41-10 y^{2}+9 y^{4}\right), \quad \theta-\theta_{2 M}=\frac{1}{1440}\left(1-y^{2}\right)\left(3-2 y^{2}+3 y^{4}\right)$,

whereas if pressure work is included we obtain

$$
\begin{array}{r}
w_{2}=\frac{1}{1920 p^{6}(\cosh 2 p-\cos 2 p)^{2}}[-30 \sinh 2 p \sin 2 p+2 \sinh 2 p y \sin 2 p y \\
+6(\cosh 2 p-\cos 2 p)(\cosh 2 p y+\cos 2 p y) \\
+\sinh 2 p(1+y) \sin 2 p(1-y)+\sinh 2 p(1-y) \sin 2 p(1+y) \\
+\frac{2}{\sinh 2 p+\sin 2 p}\left(14 \sinh 2 p \sin 2 p-3 \sinh ^{2} 2 p-3 \sin ^{2} 2 p\right) \times \\
+\frac{(\sinh p(1+y) \cos p(1-y)+\sinh p(1-y) \cos p(1+y)}{+\cosh p(1+y) \sin p(1-y)+\cosh p(1-y) \sin p(1+y))} \\
+\frac{(\sinh 2 p+\sin 2 p}{8}(\cosh 2 p-\cos 2 p)(\sinh 2 p-\sin 2 p) \times \\
\theta_{2}-\theta_{2 M}=\frac{1+y) \sin p(1-y)+\sinh p(1-y) \sin p(1+y))]}{480 p^{4}(\cosh 2 p-\cos 2 p)^{2}(\sinh 2 p+\sin 2 p)}\left[(\sinh 2 p-\sin 2 p)^{3}\right.
\end{array}
$$



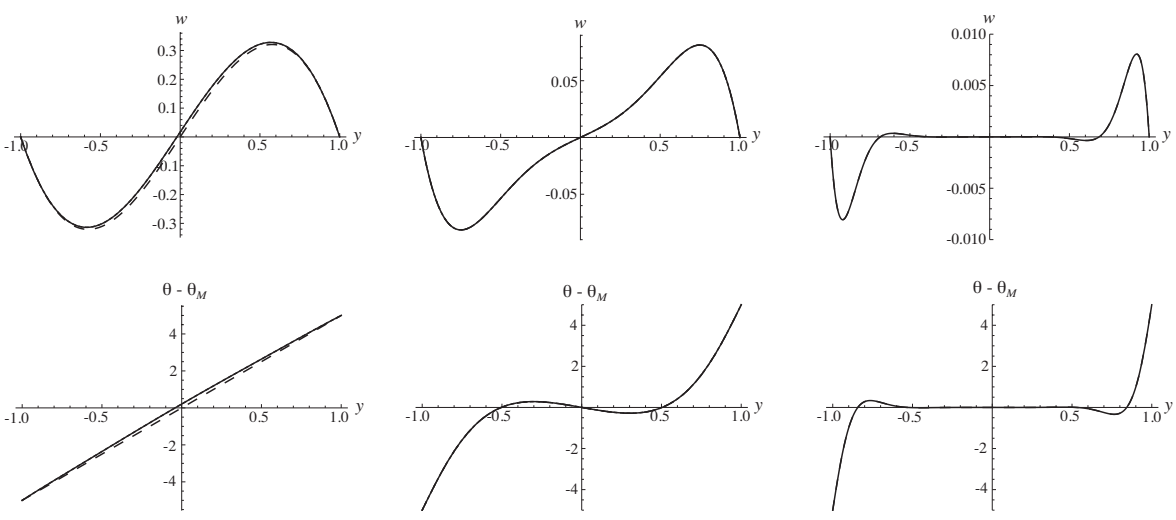

Fig. 5 Velocity and temperature profiles (relative to mean of wall temperatures) for free convection with dimensionless temperature difference $\Theta=10$ between walls. Left: excluding pressure work. Centre: with $N=400$. Right: with $N=4 \times 10^{4}$. Long-dashed curves: expansions to $O(\Theta)$. Solid curves: expansions to $O\left(\Theta^{2}\right)$. Short-dashed curves: numerical solutions (although these are indistinguishable from expansions to $O\left(\Theta^{2}\right)$ in every case).

$$
\begin{aligned}
& -8 \sinh 2 p \sin 2 p(\sinh 2 p-\sin 2 p)+8(1-\cosh 2 p \cos 2 p)(\sinh 2 p+\sin 2 p) \\
& -4 \cosh 2 p y \cos 2 p y(\sinh 2 p+\sin 2 p) \\
& -6(\cosh 2 p-\cos 2 p)(\sinh 2 p+\sin 2 p)(\cosh 2 p y-\cos 2 p y) \\
& +2(\sinh 2 p+\sin 2 p)(\cosh 2 p(1+y) \cos 2 p(1-y)+\cosh 2 p(1-y) \cos 2 p(1+y)) \\
& -\left(14 \sinh 2 p \sin 2 p-3 \sinh ^{2} 2 p-3 \sin ^{2} 2 p\right) \times \\
& \quad(\cosh p(1+y) \sin p(1-y)-\sinh p(1+y) \cos p(1-y) \\
& \quad+(\cosh p(1-y) \sin p(1+y)-\sinh p(1-y) \cos p(1+y)) \\
& +4(\cosh 2 p-\cos 2 p)(\sinh 2 p-\sin 2 p) \times \\
& \quad(\cosh p(1+y) \cos p(1-y)+\cosh p(1-y) \cos p(1+y))] .
\end{aligned}
$$

Effects of viscous dissipation (i.e. differences between $O\left(\Theta^{2}\right)$ and $O(\Theta)$ profiles) are only visible in Figure 5 in the case without pressure work, and are rather small even there; the fluid is warmed, and there is a consequent increase in upward velocity (or decrease in velocity where it is downward). The magnitude of these effects may be measured by considering temperature and velocity at the mid-plane $y=0$ : according to the numerical solution, $\theta-\theta_{M}=0.2084$ and $w=0.0170$ at $y=0$ for the case in which pressure work is excluded, with $\Theta=10$. These values decrease as $N$ increases when pressure work is included, making the profiles more nearly antisymmetric as pressure work becomes more dominant.

In order to satisfy the Boussinesq approximation, we require $\Theta$ to be small compared to $N$, so that temperature variations are small compared to absolute temperatures. This is satisfied in all the cases illustrated in Figure 5 (with $N$ 
being arbitrary where pressure work is excluded), although the large values of $N$ do imply rather large duct widths.

\subsection{Free convection with fixed wall heat fluxes}

We seek solutions of (20) and (21) with boundary conditions (25), both for the case of temporal heating $(\gamma=0)$ and for streamwise heating $(\eta=0)$, although we shall see below that for the latter case no solution exists with the equal and opposite wall heat fluxes represented by (25).

\subsubsection{Temporal heating}

We expand the velocity, temperature and heating rate in powers of the wall heat flux $q$ :

$$
\begin{aligned}
w & =w_{0}+q w_{1}+q^{2} w_{2}+\ldots, \\
\theta & =\theta_{0}+q \theta_{1}+q^{2} \theta_{2}+\ldots, \\
\eta & =\eta_{0}+q \eta_{1}+q^{2} \eta_{2}+\ldots .
\end{aligned}
$$

As in previous cases, we have $w_{0} \equiv 0$. At $O(q)$, equations (53) and (54) apply, but with boundary conditions

$$
w_{1}(-1)=0, w_{1}(1)=0, w_{1}^{\prime}(1)-w_{1}^{\prime}(-1)=0, w_{1}^{\prime \prime \prime}(-1)=-1, w_{1}^{\prime \prime \prime}(1)=-1 .
$$

The solutions are the same antisymmetric profiles (apart from constant multipliers) as were found for free convection driven by unequal wall temperatures:

$$
w_{1}=\frac{1}{6} y\left(1-y^{2}\right), \quad \theta_{1}=y
$$

if pressure work is excluded, or

$$
w_{1}=\frac{\sinh p(1+y) \sin p(1-y)-\sinh p(1-y) \sin p(1+y)}{2 p^{3}(\sinh 2 p+\sin 2 p)}
$$

with

$$
\theta_{1}=\frac{\cosh p(1+y) \cos p(1-y)-\cosh p(1-y) \cos p(1+y)}{p(\sinh 2 p+\sin 2 p)}
$$

including pressure work; compare equations $(87)-(89)$. The $O(q)$ heating rate is

$$
\eta_{1}=0
$$

even when pressure work is included: in the antisymmetric flow, any heating in the downward moving fluid is exactly compensated by cooling in the upward moving fluid. This special circumstance means that the sort of scaling arguments for velocity and heating rate that were used in the case of forced convection with insulated walls do not necessarily apply here. Starting with 

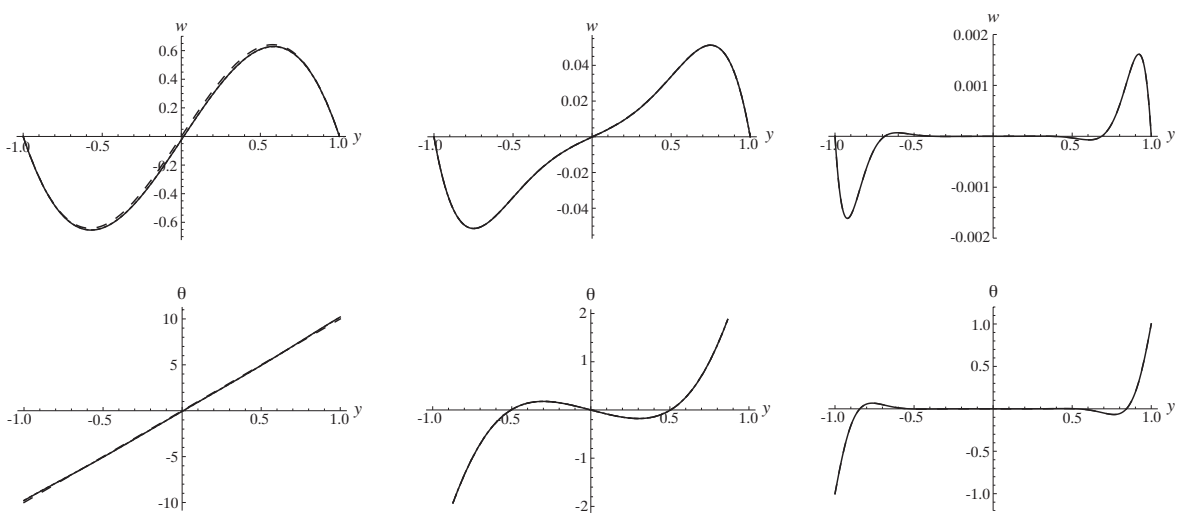

Fig. 6 Velocity and temperature profiles (relative to cross-section mean) for free convection driven by antisymmetric wall heat flux $|q|=10$. Other details as in figure 5 .

the heat-flux boundary condition $\mathrm{d}^{3} w / \mathrm{d} y^{3}=-q$, we have $w \sim q, \mathrm{~d} w / \mathrm{d} y \sim q$ and $\theta=-\mathrm{d}^{2} w / \mathrm{d} y^{2} \sim q$ if there is no pressure work or if $N$ is not large. Without pressure work, (49) applies and we have $\eta \sim q^{2}$. With pressure work and with moderate $N$, we would expect $\eta \sim-N w$ as for forced convection with insulated walls; but for our antisymmetric case the net heating by pressure work is small, so the heating rate is still governed by viscous dissipation, with the same scaling as in the absence of pressure work. Similarly, in the case of large $N$ where we might expect the velocity scaling to be $w \sim \eta / N$ as for forced convection with insulated walls, the present antisymmetric boundary conditions yield $w \sim 0$ except in boundary layers of thickness $\delta \sim N^{-1 / 4}$ adjacent to the walls. Within these boundary layers, $w \sim q \delta^{3 / 4} \sim q N^{-3 / 4}$, $\mathrm{d} w / \mathrm{d} y \sim q N^{-1 / 2}$ and $\theta \sim q N^{-1 / 4}$; inserting the scaling for $\mathrm{d} w / \mathrm{d} y$ into (49) suggests that $\eta \sim q^{2} N^{-1} \delta \sim q^{2} N^{-5 / 4}$, although we shall in fact find that $\eta \sim q^{2} N^{-1}$. The scalings for $w$ and $\theta$ in the boundary layer are in accord with the large- $p$ limits of the $O(q)$ velocity and temperature near $y= \pm 1$ from equations (99) and (100), i.e. $w \sim\left(q / 2 p^{3}\right) \sin p(1 \mp y), \theta \sim(q / p) \cos p(1 \mp y)$.

At $O\left(q^{2}\right)$ we need to solve (61) and (62) with boundary conditions

$$
w_{2}(-1)=0, w_{2}(1)=0, w_{2}^{\prime}(1)-w_{2}^{\prime}(-1)=0, w_{2}^{\prime \prime \prime}(-1)=0, w_{2}^{\prime \prime \prime}(1)=0 .
$$

Excluding pressure work, we find

$w_{2}=-\frac{1}{60480}\left(1-y^{2}\right)^{2}\left(15+10 y^{2}-9 y^{4}\right), \quad \theta_{2}=\frac{2}{945}-\frac{1}{360}\left(1-y^{2}\right)^{2}\left(1+3 y^{2}\right)$,

with heating rate

$$
\eta_{2}=\frac{1}{45}
$$


If pressure work is included,

$$
\begin{aligned}
w_{2}=\frac{1}{480 p^{8}(\sinh 2 p+\sin 2 p)^{2}}\left[-6\left(\sinh ^{2} 2 p+\sin ^{2} 2 p+3 \sinh 2 p \sin 2 p\right)\right. \\
+6(\cosh 2 p-\cos 2 p)(\cosh 2 p y+\cos 2 p y)+2 \sinh 2 p y \sin 2 p y \\
+\sinh 2 p(1+y) \sin 2 p(1-y)+\sinh 2 p(1-y) \sin 2 p(1+y) \\
+8 \sinh 2 p(\cosh p(1+y) \sin p(1-y)+\cosh p(1-y) \sin p(1+y)) \\
+8 \sin 2 p(\sinh p(1+y) \cos p(1-y)+\sinh p(1-y) \cos p(1+y))]
\end{aligned}
$$

$$
\begin{aligned}
& \theta_{2}=\frac{1}{60 p^{6}(\sinh 2 p+\sin 2 p)^{2}}[-3(\cosh 2 p-\cos 2 p)(\cosh 2 p y-\cos 2 p y)-2 \cosh 2 p y \cos 2 p y \\
& +\cosh 2 p(1+y) \cos 2 p(1-y)+\cosh 2 p(1-y) \cos 2 p(1+y) \\
& +2 \sinh 2 p(\sinh p(1+y) \cos p(1-y)+\sinh p(1-y) \cos p(1+y)) \\
& -2 \sin 2 p(\cosh p(1+y) \sin p(1-y)+\cosh p(1-y) \sin p(1+y))] \text {, }
\end{aligned}
$$

with

$$
\eta_{2}=\frac{1}{20 p^{4}} \frac{(\sinh 2 p-\sin 2 p)^{2}}{(\sinh 2 p+\sin 2 p)^{2}} .
$$

The velocity and temperature profiles, to $O(q)$ and $O\left(q^{2}\right)$ and from a numerical solution, are plotted in Figure 6 for the same cases as in Figure 5: no pressure work, $N=400$ and $N=4 \times 10^{4}$. The most obvious features are the similarity to the profiles in Figure 5 for free convection with fixed wall temperatures, and the closeness of the numerical and $O\left(q^{2}\right)$ profiles to the $O(q)$ profiles: even though the net heating rate is governed by viscous dissipation $\left(\right.$ at $\left.O\left(q^{2}\right)\right)$, this has very little effect on the velocity and temperature profiles.

Our perturbation solutions yield $\eta \approx q^{2} / 45$ without pressure work and $\eta \approx q^{2} / 5 N$ when $N$ is large. In the former case the numerical solution with $q=10$ gives $\eta=2.2153$, compared to $q^{2} / 45=2.2222$. Including pressure work and taking $q=10$ and $N=400$, the numerical solution yields $\eta=0.04996$, very close to $q^{2} / 5 N=0.05$ even though $N$ is not large enough for the profiles to take the boundary-layer form. The values of $\eta$ for free convection with $q=10$ are somewhat smaller than those found for forced convection with $\lambda=10$, so the heating rates will remain realistic for a correspondingly longer time period. Temperature variations should be small compared to $N$ in order that the Boussinesq approximation be valid, and this requirement is satisfied for all cases shown in Figure 6, bearing in mind that $N$ is arbitrary when pressure work is excluded. 


\subsubsection{Streamwise heating}

We now seek to solve (21) with $\eta=0$; we do not need to consider the case in which pressure work is included explicitly, for the reasons given in relation to forced convection with streamwise heating (Section 3.2.2 above). The velocity and temperature are expanded according to (94) and(95), and the streamwise temperature gradient is expanded similarly:

$$
\gamma=\gamma_{0}+q \gamma_{1}+q^{2} \gamma_{2}+\ldots
$$

We find $w_{0} \equiv 0$, and then at $O(q)$ we need to solve

$$
\frac{\mathrm{d}^{4} w_{1}}{\mathrm{~d} y^{4}}+\gamma_{0} w_{1}=0
$$

with the boundary conditions (97). Solutions at $O(q)$ are available for each of the cases, $\gamma_{0}=0, \gamma_{0}>0$ and $\gamma_{0}<0$. Taking firstly $\gamma_{0}=0$, we find

$$
w_{1}=\frac{1}{6} y\left(1-y^{2}\right)
$$

the same $O(q)$ profile as with temporal heating. At $O\left(q^{2}\right)$ we have

$$
\frac{\mathrm{d}^{4} w_{2}}{\mathrm{~d} y^{4}}=\left(\frac{\mathrm{d} w_{1}}{\mathrm{~d} y}\right)^{2}-\gamma_{1} w_{1}-\gamma_{0} w_{2} .
$$

Substituting $\gamma_{0}=0$ and $w_{1}$ from (110) into the right-hand side of (111), and integrating once, we obtain

$$
w_{2}^{\prime \prime \prime}=\frac{1}{180}\left(5 y-10 y^{3}+9 y^{5}\right)-\frac{\gamma_{1}}{24}\left(2 y^{2}-y^{4}\right)+A,
$$

where $A$ is a constant of integration. However, we need to satisfy the boundary conditions

$$
w_{2}(-1)=0, \quad w_{2}(1)=0,
$$

which cannot simultaneously be satisfied by (112) since the undetermined constants $\gamma_{1}$ and $A$ only apply to the even part of the solution. Similar contradictions are obtained if we take $\gamma_{0}>0$ or $\gamma_{0}<0$, but we do not display the rather messy calculations required in these cases. Hence we conclude that no perturbation solution exists. Attempts to find a numerical solution of (21) with $\eta=0$ and boundary conditions (25) also fail: specifically, taking $q=10$, we have enforced the first four conditions of (25) and sought a value of $\gamma$ for which the fifth condition is satisfied. Increasing the magnitude of $\gamma$, either positive or negative, yields values of $w^{\prime \prime \prime}(1)$ that approach the required value of $-q$, but we are unable to satisfy $w^{\prime \prime \prime}(1)=-q$ for any finite $\gamma$.

The reason for the non-existence of a solution with antisymmetric heat flux conditions and only streamwise heating is easy to see. The wall heat flux conditions tend to produce an antisymmetric flow, which then generates heat by viscous dissipation. This heat is to be removed by advection, requiring a 
temperature gradient in the direction of flow: this is not possible with a supposed uniform temperature gradient and an antisymmetric flow, even allowing for the antisymmetry of the flow being perturbed by buoyancy forces due to the heat being generated.

\section{Conclusions}

We have solved equations for fully developed flow in a vertical channel, with particular attention to the effects of pressure work and viscous dissipation in the energy equation. By using perturbation expansions we have been able to separate the effects of these two processes: pressure work comes in at first order, while dissipation only appears at second order in the velocity.

If the vertical temperature variation is unconstrained, pressure work simply results in cooling or warming of the fluid at the adiabatic lapse rate. Among the various boundary conditions that we have considered, this case only applies when heat flux (rather than temperature) is specified at the channel walls and when streamwise temperature variations are allowed. Pressure work then plays no further role in the dynamics, so can be excluded from the energy equation once the adiabatic cooling or warming is accounted for.

It is argued by Barletta [5,6] that pressure work should in any case be excluded from the energy equation when the Boussinesq approximation is applied, although Schneider [13] insists that it should be included. The present analysis does not resolve this argument; however, by presenting solutions using the energy equation both with and without the pressure work term, it clarifies the effect of including or excluding it. The argument could in principle be resolved by comparing these solutions with results obtained either from experiments or from numerical computations using equations without any approximation.

Our perturbation expansions are in terms of dimensionless forcing parameters, $\lambda$ for forced convection or $\Theta$ or $q$ for free convection, each of which is proportional to a further dimensionless parameter $N$ as well as to the respective pressure gradient, temperature difference or heat flux that is driving the flow (see equations $(26)-(28)$ ). Now, $N$ may be considered a geometric parameter, being proportional to the fourth power of the channel width (and otherwise dependent only on supposedly fixed physical properties of the working fluid); but where pressure work is included in the energy equation, it also expresses the relative importance of pressure work and viscous dissipation. Pressure work results in temperature changes which always yield a buoyancy force opposed to the motion. However, the drivers of the motion in all cases act at the channel walls (an applied pressure gradient only appearing in the equations for fully developed flow as a wall shear stress, while free convection is driven by differences in wall temperatures or by wall heat fluxes). Thus, when $N$ is large, the pressure work (if included in the equations) can extinguish the flow except in boundary layers close to the walls of the broad channel. These boundary layers are always of dimensionless width $\sim N^{-1 / 4}$, because the (di- 
mensioned) width of the boundary layer must be independent of the channel width.

All plotted results are with the relevant dimensionless forcing parameter having magnitude 10 . The plots show that viscous dissipation has very little effect on free convection flows, whether driven by temperature differences or heat fluxes at the walls: figures 5 and 6 show very little difference between the first-order perturbation solutions and the numerical solutions of the full equations. Viscous dissipation does appear to play a greater role in forced convection, except where pressure work is included and $N$ is very large; however, it should be noted that when $N$ is not large and $\lambda= \pm 10$, conversion to dimensioned variables reveals the calculated flows to be somewhat unrealistic, or at least outside the regime where the Boussinesq approximation is justified. We have chosen the rather large magnitude for $\lambda$ to bring out the effects of pressure work and viscous dissipation, but we then require a rather large channel to prevent temperature variations being greater than the absolute temperature (which occurs if $\theta-\theta_{w}>N$, see (27)) or velocities being unrealistically large: note that the dimensioned velocity is

$$
W=\left(\frac{k T_{m}}{\mu}\right)^{1 / 2} N^{-1 / 2} w
$$

where $\left(k T_{m} / \mu\right)^{1 / 2} \approx 416 \mathrm{~m} \cdot \mathrm{s}^{-1}$ in water, but $7.7 \mathrm{~m} \cdot \mathrm{s}^{-1}$ in glycerine at a temperature of $293 \mathrm{~K}$. Thus realistic flows on a laboratory scale (with $N$ not large) would require smaller values of dimensionless pressure gradient $\lambda$, with the effects of viscous dissipation (of order $\lambda^{2}$ ) being accordingly smaller than shown in Figures $1-4$. For flows on large industrial or geophysical scales, the differences between flows predicted using the formulations with or without pressure work are greater, with viscous dissipation possibly playing a major role if the latter formulation is correct, but pressure work being totally dominant in the former case.

\section{References}

1. Kay A (2016) When is natural convection completely passive? Z Angew Math Mech 96:279-303

2. Miklavčič M (2015) Stability analysis of some fully developed mixed convection flows in a vertical channel. Z Angew Math Mech 95:982-986

3. Kuiken HK (1971) The effect of compression work on free convection in gases. J Eng Math 5:51-61

4. Turcotte DL, Hsui AT, Torrance KE, Schubert G (1974) Influence of viscous dissipation on Bénard convection. J Fluid Mech 64:369-374

5. Barletta A (2008) Comments on a paradox of viscous dissipation and its relation to the Oberbeck-Boussinesq approach. Int J Heat Mass Transfer 51:6312-6316

6. Barletta A (2009) Local energy balance, specific heats and the Oberbeck-Boussinesq approximation. Int J Heat Mass Transfer 52:5266-5270

7. Barletta A, Nield DA (2009) Mixed convection with viscous dissipation and pressure work in a lid-driven square enclosure. Int J Heat Mass Transfer 52:4244-4253

8. Barletta A, Nield DA (2009) Combined forced and free convection in a vertical porous channel: the effects of viscous dissipation and pressure work. Transp Porous Media:79:319 334 
9. Barletta A, Nield DA (2009) Effect of pressure work and viscous dissipation in the analysis of the Rayleigh-Bénard problem. Int J Heat Mass Transfer 52:3279-3289

10. Barletta A (1998) Laminar mixed convection with viscous dissipation in a vertical channel. Int J Heat Mass Transfer 41:3501-3513

11. Barletta A (1999) Heat transfer by fully developed flow and viscous heating in a vertical channel with prescribed wall heat fluxes. Int J Heat Mass Transfer 42:3873-3885

12. Barletta A, Zanchini E (1999) On the choice of reference temperature for fully-developed mixed convection in a vertical channel. Int J Heat Mass Transfer 42:3169-3181

13. Schneider W (2011) Comments on M. Miklavčič and C.Y. Wang, Completely passive natural convection, ZAMM 91/7, 601-606 (2011). Z Angew Math Mech 91:1002-1004

14. Tao LN (1960) On combined free and forced convection in channels. J Heat Transfer $82: 233-238$ 